\title{
COL2A1 Mutation in Spondylometaphyseal Dysplasia Algerian Type
}

\author{
S. Matsubayashi ${ }^{a} \quad$ M. Ikema ${ }^{\text {a }} \quad$ Y. Ninomiya ${ }^{a} \quad$ K. Yamaguchi ${ }^{\text {a }} \quad$ S. lkegawa ${ }^{b}$ \\ G. Nishimurac \\ ${ }^{a}$ Department of Orthopedic Surgery, Nagasaki Prefectural Center of Medicine and Welfare for Children, Isahaya, \\ ${ }^{b}$ Laboratory of Bone and Joint Diseases, Center for Genomic Medicine, RIKEN, Tokyo, and ' Department of Pediatric \\ Imaging, Tokyo Metropolitan Children's Medical Center, Fuchu, Japan
}

\section{Key Words}

COL2A1 - Mutation · Spondylometaphyseal dysplasia

Algerian type $\cdot$ Type II collagenopathy

\begin{abstract}
Spondylometaphyseal dysplasia Algerian type (SMD-A) is an autosomal dominant disorder that was first reported in an Algerian family by Kozlowski et al. [Pediatr Radiol 1988;18:221-226]. Kozlowski's group reported a sporadic case in a 12-year-old Polish boy. They proposed SMD-A as a distinctive skeletal dysplasia and also suggested that a case of SMD reported by Schmidt et al. [J Pediatr 1963;63:106112] might have had the same disorder. Afterwards, however, no additional report has emerged to date. In addition, the question whether SMD-A belongs to type II collagenopathy (a group of disorders due to a heterozygous mutation of COL2A1) has been continuously under debate. Here we report a 7-year-old Japanese boy with a heterozygous missense mutation in COL2A1, 2582G $>$ T (Gly861Val), whose phenotype matched that of SMD-A. Our observation supports the hypothesis that SMD-A is a variant of type II collagenopathy.

Copyright $\odot 2013$ S. Karger AG, Basel
\end{abstract}

\section{KARGER}

E-Mail karger@karger.com

www.karger.com/msy
Spondylometaphyseal dysplasia (SMD) is a heterogeneous group of heritable skeletal dysplasias characterized by dysplastic development of the spine and generalized metaphyseal modifications of the tubular bones. Aside from the most common SMD Kozlowski type (OMIM 184252) and the second most common SMD corner fracture type (OMIM 184255), there are several rare subtypes and numerous unclassifiable cases of SMD. SMD Algerian type (SMD-A; OMIM 184253) is a rare subtype that is referred to as SMD with severe genu valgum in the '2010 Revision of Nosology and Classification of Genetic Skeletal Disorders' [Warman et al., 2011].

SMD-A is an autosomal dominant disorder, and the phenotypic definition is derived from 5 affected sibs in an Algerian family and a sporadic case of a Polish boy [Kozlowski et al., 1988; Rybak et al., 1991]. Rybak et al. [1991] also suggested that a case of SMD published by Schmidt et al. [1963] might have had the same disorder. Individuals with SMD-A manifest with short trunk and severe genu valgum. Myopia may be a syndromic component. The radiological hallmarks of SMD-A include moderate platyspondyly particularly with dorsal vertebral flattening, short ilia with narrow greater sciatic notches and generalized metaphyseal dysplasia of the long bones. The 
metaphyseal changes are most conspicuous in the hip and knee, and are associated with coxa vara and severe genu valgum. The short tubular bones are mildly affected. The epiphyses of the tubular bones are said to be normal.

However, no additional cases have been reported since the 2 reports by Kozlowski's group. In addition, the question whether SMD-A represents a distinctive clinical entity or falls into the phenotypic range of type II collagenopathy (a group of disorders caused by a heterozygous mutation of COL2A1) has been continuously under debate. Attention has been paid to the similarity between SMD-A and spondyloepimetaphyseal dysplasia Strudwick type (SEMD-S, a variant of type II collagenopathy; OMIM 184250), aside from 'normal epiphyses' in SMDA. Here we report a 7-year-old Japanese boy, whose phenotype matched that of SMD-A and who was found to have a heterozygous missense mutation, $2582 \mathrm{G}>\mathrm{T}$ (Gly861Val) in COL2A1.

\section{Clinical Report}

The patient is a Japanese boy born to healthy, nonconsanguineous parents. Two older sisters were healthy. Polyhydramnios was noted during pregnancy. The boy was delivered vaginally at 38 weeks and 2 days of gestation. Birth length was $40.0 \mathrm{~cm}(-4.3 \mathrm{SD})$, and weight was $2,838 \mathrm{~g}(-0.5 \mathrm{SD})$. A cleft of the soft palate and micrognathia were noted. He had difficulties in sucking and required tube feeding. Generalized skeletal changes were noted, and he was diagnosed with a chondrodystrophy. He underwent fundoplasty and gastrostomy for the management of gastroesophageal reflux at age 7 months. The cleft of the soft palate was corrected by operative surgery at age 1 year, which facilitated oral food intake. Early psychomotor development was not significantly affected, and he began to walk at the age of 2 .

At age 7, he was referred to us because of short stature and severe deformity of the legs. His height was $87.6 \mathrm{~cm}(-7.2 \mathrm{SD})$ and weight was $14.3 \mathrm{~kg}(-2.3 \mathrm{SD})$. His facies was unremarkable. Hyperlordosis with minimal scoliosis and short trunk were present. Severe genu valgum was evident. Ophthalmologic examination showed myopia. He had a hearing impairment with bilateral ear canal stenosis. His Wechsler Intelligence Scale for Children was scored as 70 . Routine blood and urinary examinations yielded normal results. Radiological examination showed a normal skull, and there was no C1/C2 instability. The spine showed platyspondyly with dorsal vertebral flattening. The ilia were broad and short with narrow greater sciatic notches. The iliac crests and acetabula appeared irregular. The proximal femoral epiphyses were mottled together with horizontal clefts. Coxa vara and short femoral necks were noted. The metaphyses of the long bones manifested irregular radiolucencies intermingled with radiodensities and were in part fragmented. Genu valgum was predominantly striking on the left (fig. 1). The short tubular bones were minimally affected. He underwent a bilateral valgus proximal femora osteotomy at age 8 . Thereafter, ossification of the proximal femoral epiphyses has somewhat progressed (fig. 2).

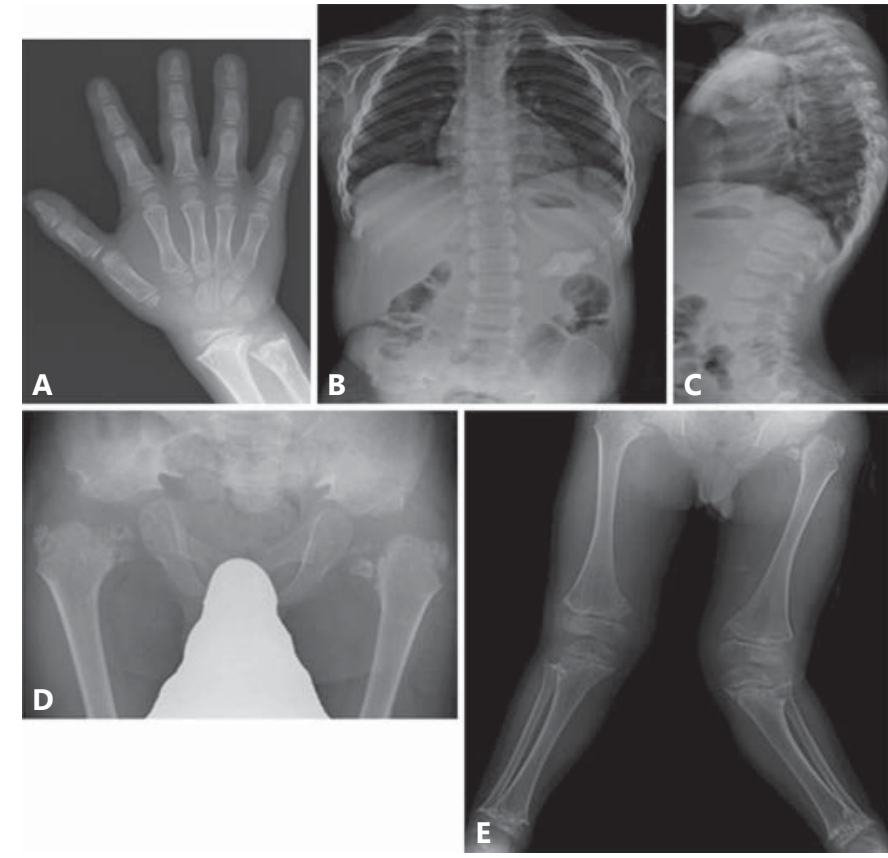

Fig. 1. Radiographs of the patient at the age of 7 years and 11 months. A Hand A-P. Short tubular bones were minimally affected. B, C Spine A-P and lateral. Moderate platyspondyly, minimal scoliosis and hyperlordosis. D Pelvis A-P. Small pelvis, shortening of the sacroiliac notches, flat and irregular acetabulum and granular texture of the capital femoral epiphysis with coxa vara. E Lower extremities. Severe metaphyseal change and valgus deformity of the knees.

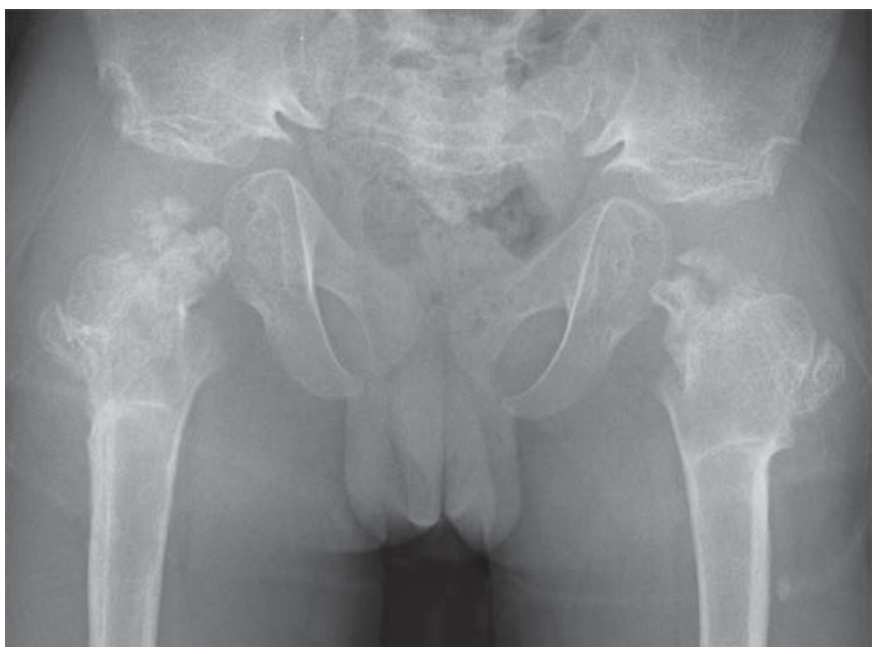

Fig. 2. Radiographs of the patient at the age of 9 years and 10 months. Pelvis A-P. The ossification of the proximal femoral epiphyses progressively improves. 


\section{Molecular Analysis}

Blood samples were obtained from the patient with informed consent, and genomic DNA was extracted using standard procedures. The entire coding regions of COL2A1, along with flanking intronic regions, were amplified by PCR using previously described primer sets [Williams et al., 1992]. PCR was performed with the exTaq system (Takara Shuzo, Ohtsu, Japan), according to the manufacturer's instructions. Sequencing was performed using an ABI 3700 automated sequencer and BigDye Terminator Cycle Sequencing Kit (Life Technologies Japan, Tokyo, Japan).

We identified a missense mutation, c. $2582 \mathrm{G}>\mathrm{T}$ (the translation start site of NM_001844 was denoted as +1) in exon 39 of COL2A1. This mutation was assumed to substitute valine for a glycine in the triple helical domain (Gly861 Val). This mutation was not found in the Human Gene Mutation Database nor SNP database. Also, it was not found in 100 Japanese control chromosomes.

\section{Discussion}

Here we report a heterozygous mutation of COL2A 1 in a sporadic case of SMD-A. The patient initially showed spondylo-(epi)-metaphyseal dysplasia (spondylo-metaphyseal dysplasia with mild dysplasia of the proximal femoral epiphyses); however, the abnormal ossification of the femoral heads improved with age. The current phenotype fits well with that of SMD-A. Our experience has shown that SMD-A may be a variant of type II collagenopathy or the same as SEMD-S.

The similarity between SMD-A and SEMD-S has attracted attention among skeletal dysplasia experts for a long period of time. Both disorders have many findings in common, including dorsal flattening of the vertebral bodies, iliac hypoplasia, extensive metaphyseal dysplasia of the long bones, and very mild affliction of the short tubular bones. The difference was thought only to be the presence or absence of dysplasia of proximal femoral epiphyses.

Although SEMD-S is widely believed to have a 'spondylo-epi-metaphyseal' pattern of skeletal changes, the entity was first described as a disorder with a 'spondylometaphyseal' pattern [Murdoch and Walker, 1969]. They entitled the first delineation of SEMD-S as a 'new' form of spondylometaphyseal dysplasia. This work was followed by an additional report by Bailey [1973], who proposed the disease name Murdock's spondylometaphyseal dysostosis. Bailey also suggested that Murdock's spondylometaphyseal dysostosis might be the same as Schmidt's spondylometaphyseal dysostosis [Schmidt et al., 1963]. However, Anderson et al. [1982] renamed the disorder as SEMD-S in their series of 14 affected individuals with similar features, including Murdock and Walker's original case. At that time, Anderson et al. [1982] chose to name the phenotype after the family name of the original patient. Thereafter, the diagnostic label of SEMD-S has tended to be given to patients with a 'spondylo-epiphyseal pattern' of bone changes associated with more or less metaphyseal alterations. Thereafter, the initial nosology of Murdock type SMD has been generally forgotten.

The nosological history of SEMD-S was exquisitely discussed by Walter et al. [2007]. The author's recent work, focusing on COL2A1-associated skeletal dysplasias with predominant metaphyseal involvement, shed a light on the early contribution by Murdoch and Walker [1969], and Bailey [1973]. In fact, the manifestations of Walter's patients 2 and 3 are identical to those of the present patient. Yet, the former patients presented with genu varum, while the latter had genu valgum. Bailey stated that the femoral head showed a grossly retarded ossification in the early age, but it began to fill from numerous ossific centers. The radiological course resembled that of the present patient. Some cases illustrated as examples of SEMD-S exhibit an almost normal ossification of the proximal femoral epiphyses [Lachman, 2007]. Finally, a patient in the second case report on SMD-A had flaky ossifications of the femoral heads [Rybak et al., 1991]. Hence, it is rational to lump SEMD-S and SMD-A together on clinical and radiological grounds.

COL2A 1 mutations create a multiplicity of clinical entities collectively termed type II collagenopathy. The present patient had a missense mutation, c. $2582 \mathrm{G}>\mathrm{T}$, which predicts substitution of valine for glycine in the triple helical domain of type II procollagen. It is well known that single nucleotide transitions that change an obligatory glycine residue in the Gly-X-Y triplet repeats to another bulkier amino acid are pathogenic. This pattern of mutations has been identified in individuals with SEMD-S, as is seen in a more common phenotype termed hypochondrogenesis/spondyloepiphyseal dysplasia congenita [Nishimura et al. 2005]. The molecular fact in the present patient warranted the proposal that SMD-A and SEMD-S reflect the phenotypic variations of essentially the same disorder.

\section{Acknowledgement}

This work was supported by Research on Intractable Diseases, Health and Labor Science Research Grants, H23-Nanchi-Ippan-123. 


\section{References}

Anderson CE, Sillence DO, Lachman RS, Toomey $\mathrm{K}$, Bull M, et al: Spondylometepiphyseal dysplasia, Strudwick type. Am J Med Genet 13: 243-256 (1982).

Bailey JA: Disproportionate Short Stature, Diagnosis and Management. (Philadelphia, W.B. Saunders 1973).

Kozlowski K, Bacha L, Massen R, Ayati M, Sator S, Brahimi L: A new type of spondylo-metaphyseal dysplasia - Algerian type. Report of five cases. Pediatr Radiol 18:221-226 (1988).

Lachman R: Taybi and Lachman's Radiology of Syndromes, Metabolic Disorders and Skeletal Dysplasias pp 1069-1071, ed 5. (Philadelphia, Mosby-Elsevier 2007).
Murdoch JL, Walker BA: A 'new' form of spondylometaphyseal dysplasia. Birth Defects Orig Art Ser 4:368-370 (1969).

Nishimura G, Haga N, Kitoh H, Tanaka Y, Sonoda $\mathrm{T}$, et al: The phenotypic spectrum of COL2A1 mutations. Hum Mutat 26:36-43 (2005).

Rybak M, Foley TP, Kozlowski K: Spondylo-metaphyseal dysplasia Algerian type: confirmation of a new syndrome. Am J Med Genet 40: 304-306 (1991).

Schmidt BJ, Becak W, Becak ML, Soibelman I, da Queiroz AS, et al: Metaphyseal dysostosis. Review of literature; study of a case with cytogenetic analysis. J Pediatr 63:106-112 (1963).
Walter K, Tansek M, Tobias ES, Ikegawa S, Coucke P, et al: COL2A1-related skeletal dysplasias with predominant metaphyseal involvement. Am J Med Genet A 143:161-167 (2007).

Warman ML, Cormier-Daire V, Hall C, Krakow D, Lachman R, et al: Nosology and classification of genetic skeletal disorders: 2010 Revision. Am J Med Genet A 155A:943-968 (2011).

Williams CJ, Harrison DA, Hopkinson I, Baldwin CT, Ahmad NN, et al: Detection of sequence variants in the gene for human type II procollagen (COL2A1) by direct sequencing of polymerase chain reaction-amplified genomic DNA. Hum Mutat 1:403-416 (1992). 